\title{
Neutropenia febril en niños con cáncer en un centro asistencial en Santiago, Chile
}

\author{
Marcela Arnello L., Juan A. Quintana B. y Pamela Barraza C.
}

\section{Febrile neutropenia in children with cancer in a medical center of Santiago, Chile}

Febrile neutropenia in cancer patients is a common complication associated to chemotherapy and can be the first manifestation of a potentially lethal infection. In order to adapt our clinical practice to national clinical guidelines, we performed a retrospective review of clinical charts of all children admitted to the Pediatric Unit of Clinica Davila, from May 1, 2003 to December 31, 2004, with cancer, fever and neutropenia. A total of 57 febrile neutropenic episodes occurred in 25 children and in 44\% (25/57) of them an infectious focus could be identified; respiratory infections were the most common (11/25). A microorganism could be identified in $16 / 57$ (x\%) of episodes, e.g. 12 recovered from blood cultures (42\% coagulase negative Staphylococcus, $17 \%$ group viridans Streptococci, $17 \%$ Escherichia coli and Enterococcus sp, Candida albicans y Pseudomonas aeruginosa in $8 \%$ each one), 3 from a catheter (coagulase negative Staphylococci) and 1 from a bronchoalveolar lavage (Pneumocystis jiroveci). At admission, 63\% of children received treatment with 2 antibiotics, mostly ceftazidime and amikacin (39\%) and $21 \%$ received vancomycin. In $37 \%$ of patients therapy required adjustment due to a poor response. Fifty six episodes had a favorable resolution with one patient diying of septic shock. Frequency of microorganism detection, the distribution of species recovered and the favorable resolution are comparable to that reported from industrialized countries.

Key words: pediatrics; febrile neutropenia; infection; cancer.

Palabras claves: pediatría; neutropenia febril; infección; cáncer.

\section{Introducción}

$\mathrm{L}$ a neutropenia en pacientes oncológicos es una complicación frecuente de la quimioterapia. La presencia de fiebre en pacientes neutropénicos con terapia inmunosupresora se considera una emergencia médica dada su alta morbi-mortalidad. A pesar del importante progreso en el manejo y resultado de los episodios de neutropenia febril (NF) en los pacientes con cáncer, la infección bacteriana continúa siendo la principal causa de morbilidad y mortalidad en estas circunstancias. Por esta razón, el diagnóstico y tratamiento de estos episodios debe ser precoz, con inicio inmediato de terapia antimicrobiana, empírica y de amplio espectro. Su manejo exige de la categorización de cada episodio en alto o bajo riesgo de adquirir una infección bacteriana invasora (IBI), concepto que ha surgido en la década de los noventa como consecuencia de una mejor comprensión de la $\mathrm{NF}^{1-3}$ y que ha permitido implementar estrategias diferenciadas, apropiadas a cada episodio según el riesgo que éste implique. En consecuencia, las intervenciones diagnósticas y terapéuticas en la NF han experimentado importantes modificaciones.
Comprender que existen episodios de NF de alto riesgo para desarrollar infecciones invasoras, de presentar complicaciones graves y de fallecer durante los episodios de $\mathrm{NF}^{1}$, ha permitido mejorar dramáticamente la sobrevida de estos pacientes, desde una mortalidad atribuible a infecciones bacterianas de $90 \%$ en la década de los sesenta, a menos del $10 \%$ en la década de los noventa ${ }^{4}$.

No obstante, aún existen importantes dificultades terapéuticas debidas a variaciones en el tiempo del espectro etiológico, la utilización de quimioterapia con mayor efecto inmunosupresor y un continuo incremento de la resistencia a antimicrobianos.

El espectro de los microorganismos causantes de las infecciones en pacientes neutropénicos ha cambiado a través de los años, evolucionando desde un predominio de bacilos gramnegativos en la década de los setenta y principios de los ochenta, a una preeminencia de cocáceas grampositivas en los noventa ${ }^{5}$. Publicaciones recientes muestran este importante aumento de las bacterias grampositivas, siendo cercano al 60 a $70 \%$ de las infecciones microbiológicamente documentadas $^{6}$. No obstante la tendencia anterior, existen variaciones etiológicas regionales, reportándose
Clínica Dávila, Santiago, Chile. Servicio de Pediatría.

Recibido: 18 de enero 2006 Aceptado: 28 junio 2006

Correspondencia a: Marcela A. Arnello Lechuga. marcearnello@yahoo.com 
un aumento en la incidencia de bacteriemias causadas por bacilos gramnegativos en algunos centros, el que llega incluso a proporciones similares en series europeas ${ }^{7}$. En estudios recientes efectuados en Chile, las etiologías más frecuentemente encontradas fueron Escherichia coli (26\%), Staphylococcus aureus (20\%), Staphylococcus coagulasa negativa (18\%), Klebsiella pneumoniae (10\%) y Pseudomonas aeruginosa $(5 \%)^{8,9}$. Se ha atribuido esta modificación en el patrón epidemiológico de las infecciones a distintos factores, entre los cuales podemos mencionar: el uso de antibacterianos profilácticos orales contra bacilos gramnegativos endógenos; el uso de catéteres venosos centrales de duración prolongada; y el daño de la mucosa oral y gastrointestinal producido por la toxicidad de altas dosis de quimioterapia, que favorece la translocación de microorganismos, antes inhabituales (como Streptococcus sp y Candida $\mathrm{sp})^{5,10}$ al torrente sanguíneo.

Un adecuado conocimiento de la epidemiología microbiana propia de cada institución es esencial para poder definir las pautas de manejo empírico de estos pacientes. Con el propósito de adecuar nuestra práctica clínica a las evidencias nacionales reunidas hasta hoy, nos planteamos revisar la experiencia institucional de los episodios de NF en pacientes pediátricos.

Objetivo de esta revisión fue describir y analizar los aspectos clínicos y microbiológicos de los episodios de NF en niños con cáncer atendidos en nuestro centro hospitalario, con el fin de conocer la realidad actual de una clínica privada y, a futuro, estandarizar el estudio y manejo de ellos, asumiendo los protocolos del Programa Infantil de Drogas Antineoplásicas-PINDA.

\section{Pacientes y Métodos}

Revisamos los registros clínicos de todos los pacientes pediátricos oncológicos, bajo 15 años de edad, que ingresaron a la Unidad de Pediatría de Clínica Dávila (UPCD), en un periodo de 19 meses $\left(1^{\circ}\right.$ de mayo 2003 - 31 de diciembre 2004), con el diagnóstico de NF.

Definiciones. Neutropenia: recuento de neutrófilos (incluye segmentados, baciliformes, juveniles, mielocitos y promielocitos) en sangre periférica inferior o igual a 500 células/ $\mathrm{mm}^{3}$.

Fiebre: presencia de temperatura axilar $\geq 38,5^{\circ} \mathrm{C}$ o dos registros térmicos $\geq 38^{\circ} \mathrm{C}$ en un período de dos horas.

Falta de respuesta a tratamiento inicial (evolución desfavorable). Se definió en base a parámetros clínicos (inestabilidad hemodinámica, fiebre persistente, nuevo foco) y de laboratorio (PCR cuantitativa en valores estacionarios o en ascenso, persistencia de cultivos positivos después de 72 horas).

Se consignaron en planillas individuales datos bio- demográficos, enfermedad de base, fecha de diagnóstico, tratamiento quimioterápico, número de episodios de NF por paciente, hallazgos clínicos (temperatura y presión arterial de ingreso, signos/síntomas sugerentes de infección pulmonar, cutánea, gastrointestinal y otros, signos inflamatorios en el sitio de inserción/ trayecto del catéter intravascular), hemograma con recuento diferencial de leucocitos al ingreso, tratamiento antimicrobiano empírico utilizado y sus modificaciones, microorganismos aislados de sangre y otros líquidos orgánicos normalmente estériles, duración de la neutropenia y de la hospitalización, uso de antimicóticos, de antivirales y factores estimuladores de colonias, ingreso a UCI, necesidad de ventilación mecánica y uso de aminas vasoactivas, mortalidad debida a infección.

Dada la naturaleza descriptiva de nuestro estudio y el reducido número de pacientes en la serie estudiada, solamente se tabularon los datos en tablas y gráficos, utilizando descriptores estadísticos habituales (promedio, rango, mediana) para las características clínicas y microbiológicas de los casos, descartándose la aplicación de análisis estadístico.

\section{Resultados}

Entre mayo del 2003 y diciembre del 2004, fueron internados en la UPCD 76 pacientes pediátricos con diagnóstico oncológico. Las patologías de base más frecuente fueron tumores sólidos $(52 \%)$, leucemias (39\%) y linfomas (8\%) (Tabla 1). De ellos, 25 pacientes presentaron NF, registrándose un total de 57 episodios (Tabla 2). El $48 \%$ de los pacientes presentó 1 episodio, $24 \%$ tuvo 2 episodios, $8 \%$ tuvo 3 episodios y $20 \%$ tuvo 4 o más episodios en el periodo estudiado; la moda fue de 2,3 episodios por paciente (1 a 8).

En el universo analizado $54 \%$ eran mujeres, y las edades oscilaron entre 1 y 15 años, con una mediana de 6,8 años (Tabla 3).

En las Tablas 1 y 2 se resume la importancia relativa de la NF según la patología de base del paciente y el número de episodios de NF observado por enfermedad de base, respectivamente. El diagnóstico oncológico predominante $(69 \%)$ fue leucemia linfoblástica aguda (LLA); en el $50 \%$ de estos pacientes hubo un total de 14 episodios de NF y el resto no presentó NF. En $31 \%$ de los pacientes con leucemia mieloblástica aguda (LMA), se observó un total de 6 episodios. Un paciente con linfoma de Burkitt presentó 5 episodios de NF y el resto de los pacientes con linfomas no presentó episodio alguno. Los tumores sólidos fueron la principal patología de base $(56 \%)$, seguido por leucemias (35\%) y linfomas (9\%). El sarcoma de Ewing 
y el meduloblastoma fueron los tumores sólidos más frecuentes, (25\% cada uno). La LLA se presentó en $25 \%$ de los pacientes y la LMA en $11 \%$.

En $90 \%$, el episodio de NF ocurrió en el primer año de enfermedad y, en la mayoría, a menos de 6 meses desde la fecha de diagnóstico: $(77 \%)$ y en $51 \%$ a 1 semana o menos del último ciclo de quimioterapia.

La consulta fue precoz (durante las primeras 24 horas de iniciada la fiebre) en $91 \%$ de los pacientes.

En $95 \%$ de los pacientes la enfermedad se encontraba activa (en quimioterapia endovenosa) al momento del ingreso.

Con respecto a los parámetros clínicos al ingreso, la temperatura media fue de $38,0{ }^{\circ} \mathrm{C}$; el $47 \%$ de los pacientes presentó una temperatura entre $38-39{ }^{\circ} \mathrm{C}$ y sólo $12 \%$ un pico de temperatura mayor a $39^{\circ}$. La presión arterial fue normal en $81 \%$ y $6 / 57$ casos $(11 \%)$ presentaron hipotensión arterial (Tabla 3).

Aplicando los criterios actuales utilizados por el PINDA para clasificar el riesgo de cada episodio, el $53 \%$ de los episodios de NF estudiados corresponderían a situaciones de bajo riesgo.

Se logró establecer el foco infeccioso en 25/57 (44\%) de los casos: patología respiratoria en 11/57 (19,3\%), foco cutáneo en $6 / 57$ (10,5\%) y digestivo en 4/57 (7\%); 4 pacientes $(7 \%)$ presentaron shock séptico requiriendo traslado a UCI. Seis pacientes presentaron bacteriemia. No se comprobó una causa infecciosa en $56 \%$ de los casos, ni se pudo determinar con precisión el origen de la fiebre en ellos. En la Tabla 4 se enumeran los diagnósticos de egreso y en cuántos de ellos hubo documentación microbiológica.

El agente infeccioso se identificó en $28 \%$ de los casos (16/57), con predominio de cocáceas grampositivas $(67 \%)$ y luego bacilos gramnegativos. Se aislaron 12 cepas desde sangre (Figura 1), 3 fueron aisladas de cultivos de catéter infectados con Staphylococcus coagulasa negativa y una de lavado bronquio-alveolar (Pneumocystis jirovecii).

Como terapia antimicrobiana empírica de ingreso, $63 \%$ recibió dos fármacos, siendo la combinación más utilizada ceftazidima y amikacina (39\%). Otras combinaciones utilizadas fueron: ceftazidima-vancomicina (22\%), ceftazidima-cloxacilina (17\%), cefotaximacloxacilina (17\%), ceftazidima- penicilina sódica $(3 \%)$ y cefazolina-amikacina (3\%). Triterapia recibió $32 \%$ de los pacientes, de preferencia ceftazidima más amikacina más vancomicina y ceftazidima más amikacina más cloxacilina (33\% cada una). Sólo 5\% recibió monoterapia (ceftazidima). En 21\% (21/57) de los pacientes se utilizó vancomicina al ingreso. Por falta de respuesta al tratamiento anti-infeccioso inicial, en $37 \%$ de los pacientes debió modificarse la terapia; en $72 \%$ de ellos se utilizó vancomicina combinada.

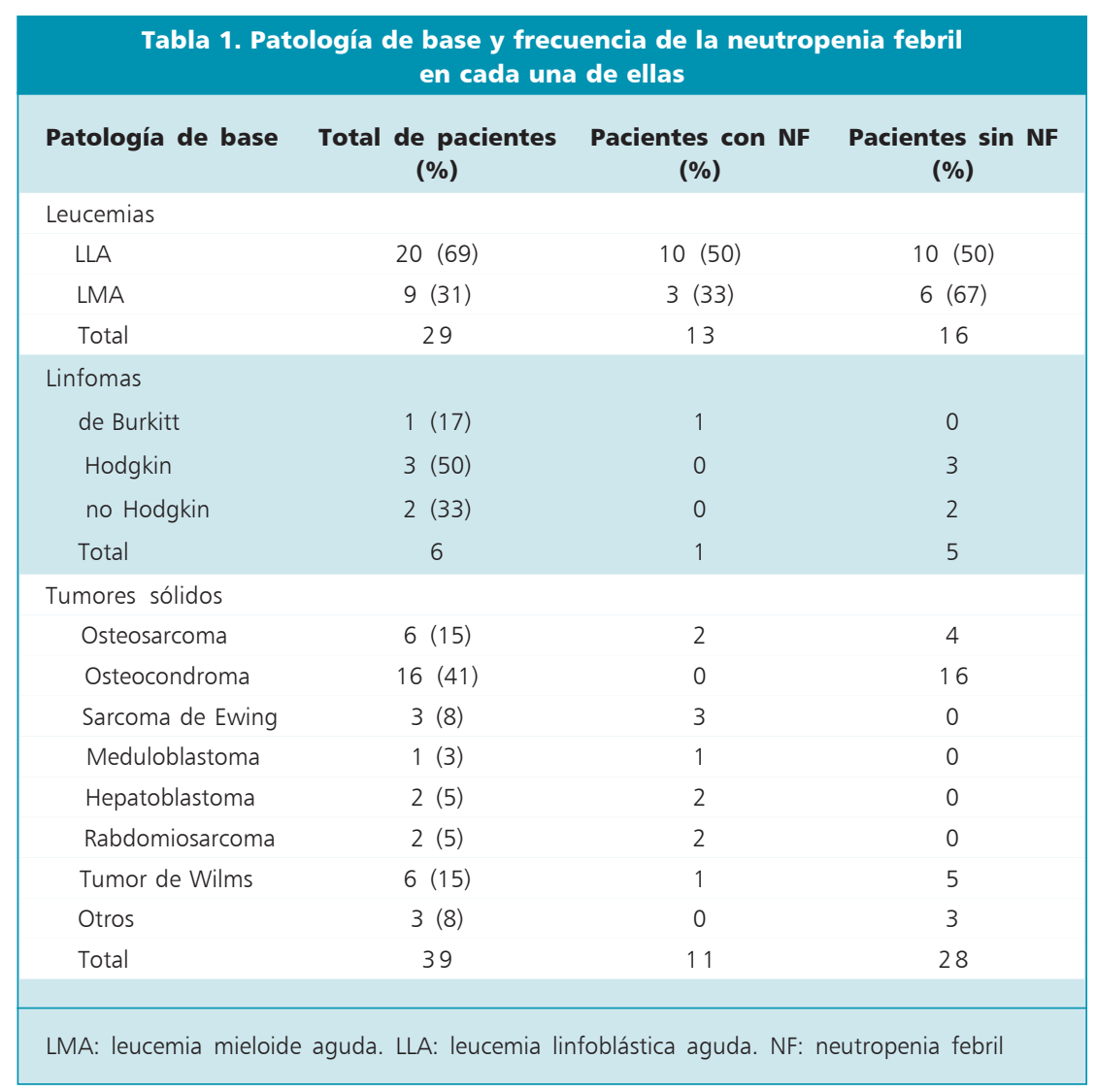

\begin{tabular}{|lcc|}
\hline \multicolumn{2}{|l|}{ Tabla 2. Episodios de neutropenia febril según patología de base } \\
\hline Patología de base & N episodios & $\%$ \\
\hline LMA & 6 & 10,5 \\
\hline LLA & 14 & 24,6 \\
\hline Tumores sólidos & 32 & 56,1 \\
\hline Linfoma de Burkitt & 5 & 8,8 \\
\hline Total & 57 & 100,0 \\
\hline
\end{tabular}

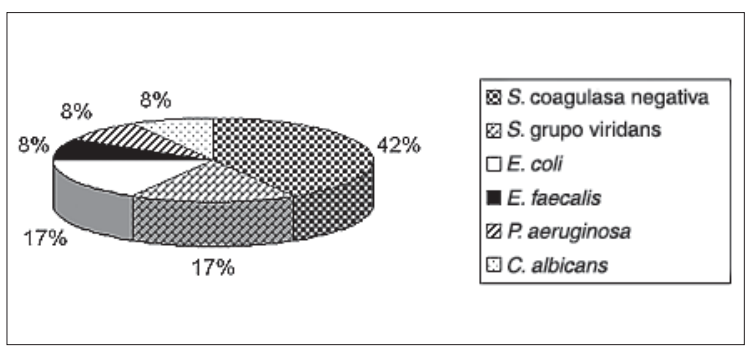

Figura 1. Microorganismos aislados en hemocultivos ( $\mathrm{n}$. 12). 


\begin{tabular}{|c|c|c|}
\hline & $\mathbf{N}^{\circ}$ pacientes & $\%$ \\
\hline \multicolumn{3}{|l|}{ Sexo } \\
\hline Masculino & 26 & 45,6 \\
\hline Femenino & 31 & 54,4 \\
\hline \multicolumn{3}{|l|}{ Edad (años) } \\
\hline Media & 6,8 & \\
\hline Rango & 1 a 15 & \\
\hline \multicolumn{3}{|l|}{ Enfermedad de base } \\
\hline Tumores sólidos & 32 & 56,1 \\
\hline Leucemias & 20 & 35,1 \\
\hline Linfomas & 5 & 8,8 \\
\hline \multicolumn{3}{|c|}{ Horas de fiebre preingreso } \\
\hline $0-24$ hrs & 52 & 91,2 \\
\hline $24-48$ hrs & 2 & 3,5 \\
\hline$>48 \mathrm{hrs}$ & 3 & 5,3 \\
\hline Total & 57 & 100,0 \\
\hline \multicolumn{3}{|l|}{ Temperatura al ingreso } \\
\hline$\leq 37^{\circ}$ & 10 & 17,5 \\
\hline $37-38^{\circ}$ & 13 & 22,8 \\
\hline$>38-39^{\circ}$ & 27 & 47,4 \\
\hline$>39-40^{\circ}$ & 7 & 12,3 \\
\hline$>40^{\circ}$ & 0 & 0 \\
\hline Total & 57 & 100,0 \\
\hline \multicolumn{3}{|c|}{ Presión arterial al ingreso } \\
\hline Hipotensión & 6 & 10,5 \\
\hline Normal & 46 & 80,7 \\
\hline Aumentada & 5 & 8,8 \\
\hline Total & 57 & 100,0 \\
\hline
\end{tabular}

Tabla 4. Diagnóstico de egreso para los 57 episodios de neutropenia febril

\begin{tabular}{|c|c|c|}
\hline Diagnóstico & $\mathbf{N}^{\circ}$ de episodios & Confirmación microbiológica \\
\hline Fiebre de origen desconocido & 26 & 0 \\
\hline IRA alta & 7 & 2 \\
\hline Bronconeumonía & 2 & 2 \\
\hline Bronconeumonía viral & 2 & 0 \\
\hline Bacteriemia & 6 & 6 \\
\hline Diarrea aguda & 4 & 0 \\
\hline Celulitis & 5 & 2 \\
\hline Infección cutánea superficial & 1 & 0 \\
\hline Shock séptico & 4 & 4 \\
\hline
\end{tabular}

Susceptibilidad in vitro. Todas las cepas de Staphylococcus coagulasa negativa fueron resistentes a oxacilina y sensibles a vancomicina. Las cepas de Streptococcus grupo viridans fueron sensibles a cefalosporinas y resistentes a penicilinas. No se aisló Enterococcus resistente a aminoglucósidos o vancomicina. Las 2 cepas de E. coli aisladas fueron resistentes a cefalosporinas de tercera generación. La cepa de $P$. aeruginosa aislada fue sensible a ceftazidima.

Terapias no anti-bacteriana. Los criterios considerados para la introducción de estas terapias fueron variables: para el uso de antimicóticos, persistencia de fiebre al séptimo día con RAN $<500$ células/ $\mathrm{mm}^{3}$ y evaluación clínica, de laboratorio y por imágenes; para el uso de antivirales, evidencia clínica o de laboratorio de enfermedad viral; para el uso de filgrastim, neutropenia grave y prolongada. La indicación fue hecha por el médico tratante.

Veintiocho por ciento recibió antimicóticos durante su hospitalización, de preferencia fluconazol (78\%). En sólo un paciente se obtuvo un hemocultivo positivo para $C$. albicans, recibiendo tratamiento con anfotericina B y luego fluconazol. En $11 \%$ se utilizó aciclovir y en $62 \%$ factor estimulador de colonias de granulocitos (filgrastim-Neupogen®). El tiempo de duración de la neutropenia fue menor o igual a 7 días en $72 \%$ de los casos, con una media de 5,4 días (rango 1-20) y la duración media de hospitalización fue de 7,2 días, con un rango de 1 a 34 días; sólo 5\% requirió una hospitalización prologada mayor a 1 mes.

Cuatro pacientes ingresaron a UCI a causa de un shock séptico, requiriendo uso de ventilación mecánica y aminas vasoactivas.

Del total de episodios, 56 tuvieron una evolución favorable. Un paciente falleció durante el período de estudio a consecuencia de un shock séptico secundario a bacteriemia por $E$. coli, determinando una mortalidad global para esta experiencia de 1,8\%.

\section{Discusión}

La NF en los pacientes oncológicos es reconocida como una de las complicaciones y limitaciones más importantes de la quimioterapia en términos de morbimortalidad, siendo las infecciones la principal consecuencia de ella. Se espera que sobre $80 \%$ de los pacientes sometidos a quimioterapia intensiva desarrollen fiebre durante los episodios de neutropenia y de ellos, en aproximadamente $50 \%$, se pesquisará una infección o ella permanecerá oculta ${ }^{6,11}$. La confirmación microbiológica es desgraciadamente insuficiente, en 20 a $30 \%$ se demuestra una infección bacteriana según distintas series ${ }^{6,8,11}$; en otro 30 a $40 \%$ se logra 
documentar clínicamente la infección y el resto de los episodios es catalogado como fiebre de origen desconocido (FOD). Los diagnósticos más frecuentemente asociados a la NF varían según los distintos grupos de riesgo, siendo bronconeumonía y sepsis los más frecuentes entre los pacientes de alto riesgo, y FOD entre los de bajo riesgo ${ }^{12}$.

En nuestro estudio se diagnosticó una infección como causa de fiebre en $44 \%$ de los episodios, porcentaje similar al reportado como promedio en la literatura médica; se documentó una etiología microbiana en más de la mitad de estos casos (16/31), lo que coincide con la mayoría de las series. En relación con los microorganismos aislados, la mayor parte de los trabajos refieren 60 a $70 \%$ de cocáceas grampositivas ${ }^{6,13}$, con un alto índice de resistencia a antibacterianos (SAMR, $S$. grupo viridans resistente a penicilina, etc). Nuestra experiencia es concordante con dichas publicaciones ya que del total de los aislados $67 \%$ correspondieron a cocáceas grampositivas y sólo $25 \%$ a bacilos gramnegativos. Es importante recalcar que todos los aislados de $S$. coagulasa negativa fueron resistentes a oxacilina y susceptibles a vancomicina y que las dos cepas de Streptococcus grupo viridans, fueron resistentes a penicilinas. Datos chilenos obtenidos en estudios prospectivos, multicéntricos, dan cuenta de los mismos agentes como causa de bacteriemias, describiéndose resistencia de $S$. aureus y Staphylococcus coagulasa negativa a cloxacilina en 44 y $55 \%$ respectivamente ${ }^{13,14}$.

El tratamiento antimicrobiano recibido por nuestros pacientes fue variable, no protocolizado. La terapia empírica más utilizada al ingreso fue una combinación de cefalosporina con actividad anti-pseudomonas (ceftazidima) y un aminoglucósido (amikacina). No se realizó una categorización en grupos de riesgo que permitiera un manejo selectivo de ellos. Nuestra Unidad Clínica de Oncología es relativamente nueva, con sólo 5 años de funcionamiento; además, durante este período trabajaron dos grupos de oncólogos, con diferentes pautas de tratamientos, lo que explicaría la diversidad observada en el estudio y manejo de los episodios.

En conclusión, es importante reforzar la necesidad una adecuada clasificación de los episodios de NF, valorando las variaciones inter-individuales, y efectuar una vigilancia microbiológica continua, que nos permita conocer la epidemiología local de las bacterias prevalentes y sus patrones de resistencia. Así se podrán definir los tratamientos empíricos más apropiados para cada episodio, lo que permitiría disminuir la morbilidad y evitar la mortalidad relacionada, otorgando además amplios beneficios económicos.

La corta duración de los episodios de NF y los bajos perfiles de resistencia observados en nuestra experiencia avalan la implementación de un manejo ambulatorio y/o el alta precoz con fármacos orales, de aquellos pacientes con episodios de NF con bajo riesgo de complicación (53\%).

Por tratarse de una revisión totalmente descriptiva, la calidad de la información no es óptima y está afectada por los sesgos propios de un estudio retrospectivo. Nuestro propósito es a corto plazo estandarizar la atención de nuestros pacientes.

\section{Conclusiones}

La proporción de episodios con causa infecciosa documentada microbiológica y clínicamente, fue concordante con la mayoría de las series publicadas, al igual que las características de las especies aisladas y la evolución favorable de esta grave complicación.

Nuestra experiencia confirma la heterogeneidad aplicada al estudio y manejo de la NF en nuestro centro. Creemos necesario protocolizar los episodios y uniformar criterios en la atención de nuestros pacientes, con el objetivo de ofrecerles un enfoque terapéutico racional, proporcionado a la gravedad de sus episodios de $\mathrm{NF}$, clasificándolos en alto y bajo riesgo de adquirir una infección bacteriana invasora. Actualmente estamos iniciando en nuestra clínica un protocolo prospectivo para la evaluación de estos pacientes, que se comenzará a aplicar en los próximos meses.

\section{Resumen}

La neutropenia febril (NF) en pacientes oncológicos es una complicación frecuente de la quimioterapia y primera manifestación de una infección potencialmente letal. Con el propósito de adecuar nuestra práctica médica a las guías clínicas vigentes en Chile, revisamos la experiencia de la Unidad de Pediatría, Clínica Dávila, entre mayo $1^{\circ}$, de 2003 y diciembre 31, de 2004. Se analizaron 57 episodios (25 pacientes); se encontró foco infeccioso en $44 \%$ (25/57) siendo respiratorio en $11 / 25$. Se identificó el agente infeccioso en $28 \%$ (16/57): 12 cepas desde sangre (42\% Staphylococcus coagulasa negativa, $17 \%$ Streptococcus grupo viridans, $17 \%$ Escherichia coli y Enterococcus sp, Candida albicans y Pseudomonas aeruginosa en $8 \%$ cada uno), 3 de cultivos de catéter ( $S$. coagulasa negativa) y 1 en lavado bronquioalveolar (Pneumocystis jiroveci). Al ingreso, $63 \%$ recibió tratamiento con 2 antibacterianos, de preferencia ceftazidima y amikacina (39\%), en $21 \%$ se utilizó vancomicina. Por falta de respuesta, en $37 \%$ 
de los pacientes se cambió la terapia. Cincuenta y seis episodios tuvieron una evolución favorable y un paciente falleció por shock séptico. La proporción de episodios con causa infecciosa documentada, los microorganismos aislados y la resolución favorable de esta grave complicación en pacientes oncológicos demuestran un nivel de diagnóstico y manejo comparable con países industrializados.

\section{Referencias}

1.- Santolaya M E. Neutropenia febril en el niño con cáncer. Conceptos actuales sobre criterios de riesgo y manejo selectivo. Rev Méd Chile 2001; 129: 1449-54.

2.- Santolaya M E, Álvarez A M, Avilés C, Becker A, Cofré J, Payá E, et al. Tratamiento selectivo de los episodios de neutropenia febril en niños con cáncer. Comité de Infectología, Programa Infantil Nacional de Drogas Antineoplásicas (PINDA). Rev Chil Infect 2004; 21: 213-22.

3.- Klaassen R J, Goodman T R, Pham B, Doyle J. Low risk prediction rule for pediatric oncology patients presenting with fever and neutropenia. J Clin Oncol 2000; 18: 1012-19.

4.- Viscoli C, Castagnola E. Treatment of febrile neutropenia: what is new?. Curr Opin Infect Dis 2002; 15:377-82.

5.- Santolaya M E, Rabagliati R, Bidart T, Payá E, Guzmán A M, Morales R, et al. Consenso: Manejo racional del paciente con cáncer, neutropenia y fiebre. Rev Chil Infect 2005; 22 (Supl 2): S79-S114.
6.- Hughes W T, Armstrong D, Bodey G P, Bow E J, Brown A E, Calandra T, et al. 2002 Guidelines for the use of antimicrobial agents in neutropenic patients with cancer. Clin Infect Dis 2002; 34: 730-51.

7.- Marcheti O, Calandra T. Infections in neutropenic cancer patients. Lancet 2002; 359: 723-5.

8.- Santolaya M E, Álvarez A M, Becker A, Cofré J, Enríquez N, O’Ryan M, et al. Prospective, multicenter evaluation of risk factors associated with invasive bacterial infection in children with cancer, neutropenia and fever. J Clin Oncol 2001; 19: 3415-21.

9.- Santolaya M E, Álvarez A M, Avilés C L, Becker A, Cofré J, Enríquez N, et al. Prospective evaluation of a model of prediction of invasive bacterial infection risk among children with cancer, fever and neutropenia. Clin Infect Dis 2002; 35: 678-83.

10.- Hormaechea A, Martínez P, Zolezzi P, Folatre I, Cruz M C, Leal N, et al. Neutropenia febril en menores con leucemia linfoblástica aguda. Rev Chil Pediatr 2004;
75: $146-52$

11.- Manterota A, Romero P, Martínez E, Villafranca E, Arlas F, Domínguez M A, et al. Neutropenia y fiebre en el paciente con cáncer. An Sist Sanit Navar 2004; 27: 3343.

12.- Rizzardini C, Espinoza X. Urgencias oncológicas. Rev Ped Elec (en línea) 2005; 2: 25-32 (Accedido 20 noviembre 2005).

13.- Payá E, Álvarez A M, Avilés C, Cofré J, Enríquez N, Salgado C, et al. Agentes causantes de infecciones del torrente circulatorio en niños con cáncer, en cinco hospitales de Santiago (1994-1998). Rev Méd Chile 2001; 129: 1297-304.

14.- Trucco O, Prado V, Díaz M C, Ojeda A y Grupo PRONARES. 24 meses de vigilancia de patógenos asociados a bacteriemias y su patrón de resistencia a antimicrobianos. Programa de vigilancia PRONARES. Libro resúmenes XVII Congreso Chileno de Infectología, Viña del Mar 9-12 de agosto 2000. Resumen P-86.

15.- Quintana J, Barraza P. Guías de práctica clínica: Neutropenia febril. Clínica Dávila Marzo 2005. 